\title{
Quasi-Static and Dynamic Ballistic Damage Mechanisms in Confined Ceramic Tiles
}

\author{
D. Sherman
}

Department of Materials Engineering, Technion, Israel Institute of Technology, Haifa 32000, Israel

\begin{abstract}
Quasi-static and dynamic damage mechanisms in alumina tiles were investigated. The tiles were under a variety of boundary conditions at the tile's edges during impact. NATO 0.3 cal. AP rounds with hardened and sharpened steel core were used. The alumina tile samples were $50 \times 50 \mathrm{~mm}^{2}$ area, the thicknesses ranging from 5 to $12 \mathrm{~mm}$. The tiles were mounted in a special confinement frame, which enabled to investigate the failure mechanisms under a large variety of boundary conditions. Steel and Spectra composite were used as backing plates, for their distinct modulus of elasticity and acoustic impedance. Three different support configurations were used: semi-infinite, thick walled, and simply supported.
\end{abstract}

\section{INTRODUCTION}

Monolithic ceramics have been evaluated for their ballistic performance and the results have led to a well-established understanding of the requirements for ceramic armor [1-6]. Several methods have been used to confine ceramic tile under compressive deformation [5-8]. In all of them the ballistic damage was reduced. It was difficult, however, to quantify the exact boundary conditions or compressive stresses during testing. We use a new, effective and simple method to generate high compressive stresses in a ceramic tile [6], which enables the study of the effect of biaxial compressive stresses on the failure mechanisms in ceramic tiles. By changing the confinement prestress level and boundary conditions, the quasi-static and the dynamic damage could be distinguished. Alımina tiles supported by semi-infinite steel and confined by steel wedges suffered no stress wave reflections due to almost identical impedance. The dynamic damage was achieved by placing the tile on Spectra ${ }^{T M}$ (Allied-Signal) composite support. Biaxial confinement of the tile significantly reduced the quasi static, but not the dynamic damage.

\section{EXPERIMENTAL PROGRAM}

\subsection{The Confinement Frame}

The design of the confinement frame, shown in Fig. 1, is fully described in [6]. The ceramic tile rests on a slightly undersized support block, and mechanically clamped at its sides by four bolted vertical wedges. The wedges and main base were each designed with a wedge angle of $15^{\circ}$, which produces a high clamping load on the tile. A torquemeter was used to control the torque applied to each of the eight bolts fastening the wedges. The tiles can thus be constrained by a high biaxial compressive stress. A $30 \mathrm{~mm}$ diameter hole was drilled in the cover plate that allowed for direct impact of the tile. Both the wedges and the cover plate where quenched and annealed to $50 \mathrm{Rc}$ in order to reduce any plastic deformation generating during the impact process.

A calibration function between the clamping torque and the biaxial compressive stresses in the tile was achieved by a set of strain gages. The final torque-stress relationship obeys $\sigma=8.4(\mathrm{t} / 5) \mathrm{T}$, where $\mathrm{T}$ is the clamping torque in $\mathrm{Nm}$ and $\mathrm{t}$ is the tile's thickness in $\mathrm{mm}$. The stresses are in $\mathrm{MPa}$. 

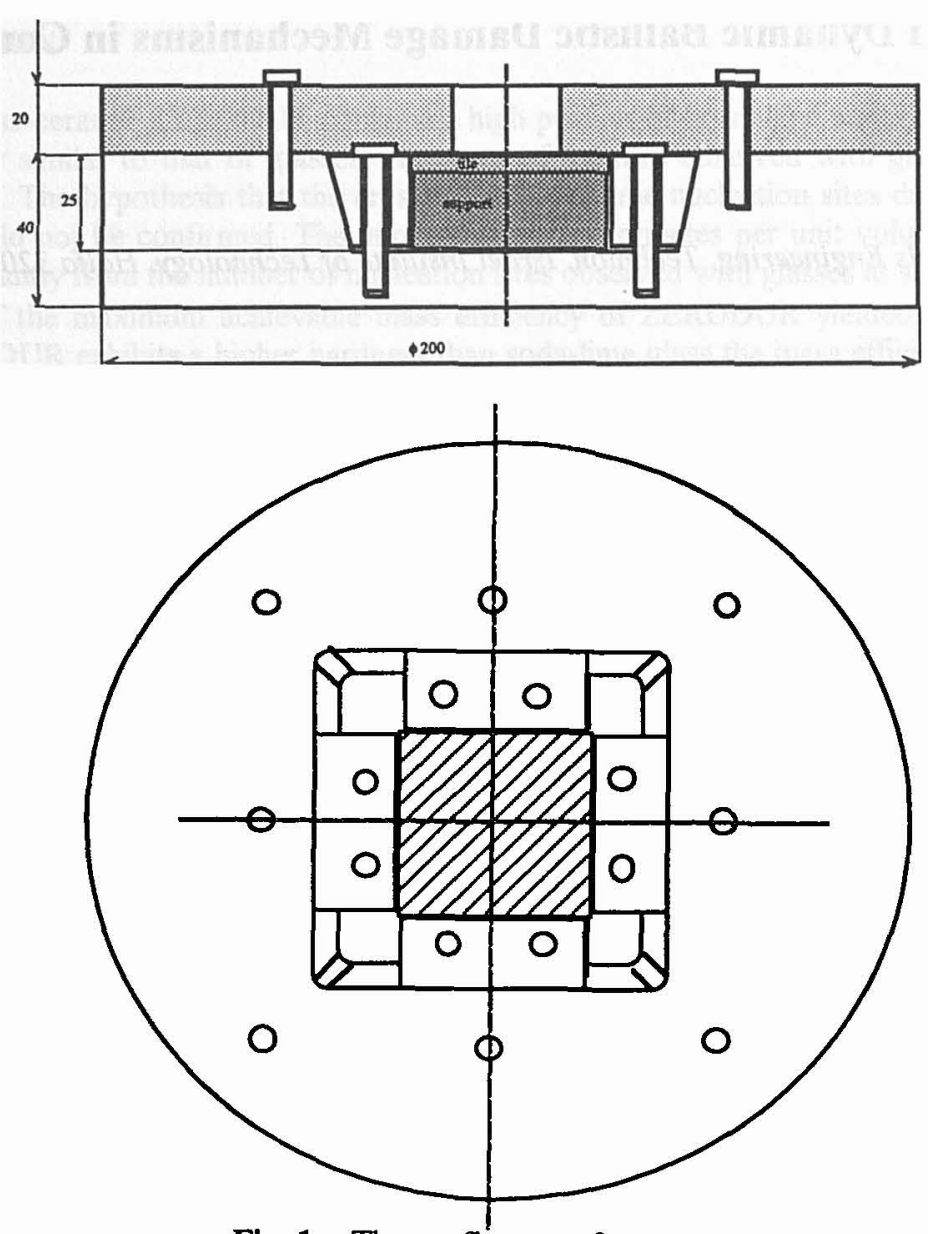

Fig. 1. The confinement frame

\subsection{Boundary conditions}

Three different confinement conditions were applied: i) the tile was rested on a support with no lateral confinement (Fig. 2a), ii) was confined by the four wedges that enforced 'zero' movement of the tile's fragments (Fig. 2b), and iii) was confined under a biaxial compressive stress of $200 \mathrm{MPa}$ (Fig. 2c).

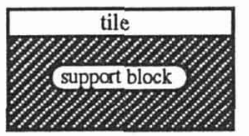

(a)

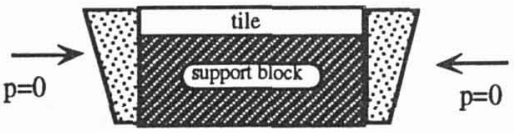

(b)

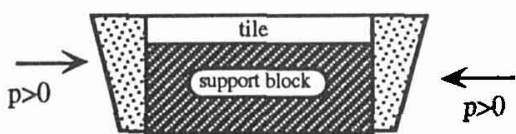

(c)

Fig. 2. The confinement conditions: no confinement (a), zero confinement (b) and confined (c).

\subsection{Tile material}

The tiles were $98 \%$ grad alumina with an average grain size of $10 \mu \mathrm{m}$ (Rami Ltd. Israel). Tile area was $50 \times 50 \mathrm{~mm}^{2}$, and the thickness ranged between 5 and $12 \mathrm{~mm}$. All the tiles were ground flat to 
avoid uncontrolled bending or stress wave reflections. A thin foil of tin $(0.15 \mathrm{~mm})$ was attached to the confined tiles' edges in order to refine the stress distribution there.

\subsection{Support configuration}

Three types of support configuration were used: the first was a semi infinite support (Fig. 3a), the second was a finite width (Fig. 3b), and the third simulated the tile as being a simply supported plate (Fig. 3c). A circular hole of $30 \mathrm{~mm}$ diameter was drilled in the latter two types of supports.

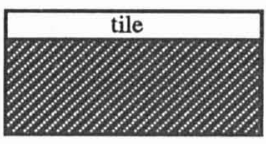

(a)

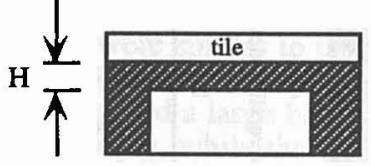

(b)

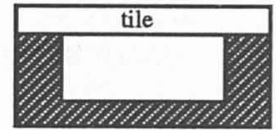

(c)

Fig. 3. Support configurations: semi infinite (a), finite width (b) and simply supported (c).

\subsection{Support materials}

4340 steel was used for the three types of support described above. Spectra composite was used for the semi-infinite support configuration. The impedance mismatch of the steel/alumina assembly is low, while it is high for alumina/Spectra assembly (polystyrene properties were taken for Spectra) Table 1.

\begin{tabular}{|c|c|c|c|c|}
\hline & $\begin{array}{l}\text { Young's } \\
\text { Modulus }\end{array}$ & Density & $\begin{array}{l}\text { Long. } \\
\text { veloc. }\end{array}$ & $\begin{array}{l}\text { Long. } \\
\text { imped. }\end{array}$ \\
\hline & $\begin{array}{c}\mathrm{E} \\
\text { [GPa] }\end{array}$ & $\begin{array}{c}\rho \\
{\left[\mathrm{Kg} / \mathrm{m}^{3}\right]}\end{array}$ & $\begin{array}{c}\mathrm{V}_{\mathrm{L}} \\
{[\mathrm{m} / \mathrm{s}]}\end{array}$ & ${ }_{\left[10^{6} \mathrm{Kg} / \mathrm{m}^{2} \mathrm{~s}\right]}^{\mathrm{Z}}$ \\
\hline Alumina & 350 & 3900 & 9870 & 42 \\
\hline 4340 steel & 210 & 7700 & 5900 & 45 \\
\hline Polystyrene & 3 & 1060 & 2350 & 2.5 \\
\hline
\end{tabular}

Table 1. Relevant physical properties of constituents [9].

\subsection{AP conditions}

Tile samples were impacted by the NATO $0.3 \mathrm{cal}$. AP rounds. The round's velocity at the target was measured to be $850 \pm 10 \mathrm{~m} / \mathrm{sec}$. The average kinetic energy of a round is $3.4 \mathrm{KJ}$.

\section{RESULTS AND DISCUSSION}

\subsection{Semi-infinite supports}

\subsubsection{Steel support}

The boundary conditions at the edges significantly affected the damage to tiles supported by steel support. All the tiles with no lateral confinement shattered to a large variety of fragments. Evidence for radial crack patterns was found on the steel supports. Tiles with zero lateral displacement experienced reduced damage. The tiles remained intact, revealing the damage mechanisms. The damage to tiles confined by biaxial compressive stresses of $200 \mathrm{MPa}$ reduced dramatically. Fig. 4 depicts the minor damage to a $10 \mathrm{~mm}$ thick tile in form of 9 partially developed radial cracks.

The maximum irreversible deflection at the steel support beneath the contact zone under various boundary conditions and tile thicknesses are shown in Fig. 5 and well demonstrate the effect of confinement.

Test results of impacted tiles supported by semi-infinite steel supports have lately been widely reported $[4,6]$. The damage was mainly quasi-static in nature, due to vanishing reflected stress waves resulted nearly identical acoustic impedance of alumina and steel (Table 1). Radial tensile cracks, shear dominated cone crack and fragmentation of the cone envelope are the major ballistic damage mechanisms. The mechanisms and sequence are affected by tile's thickness [6]. 

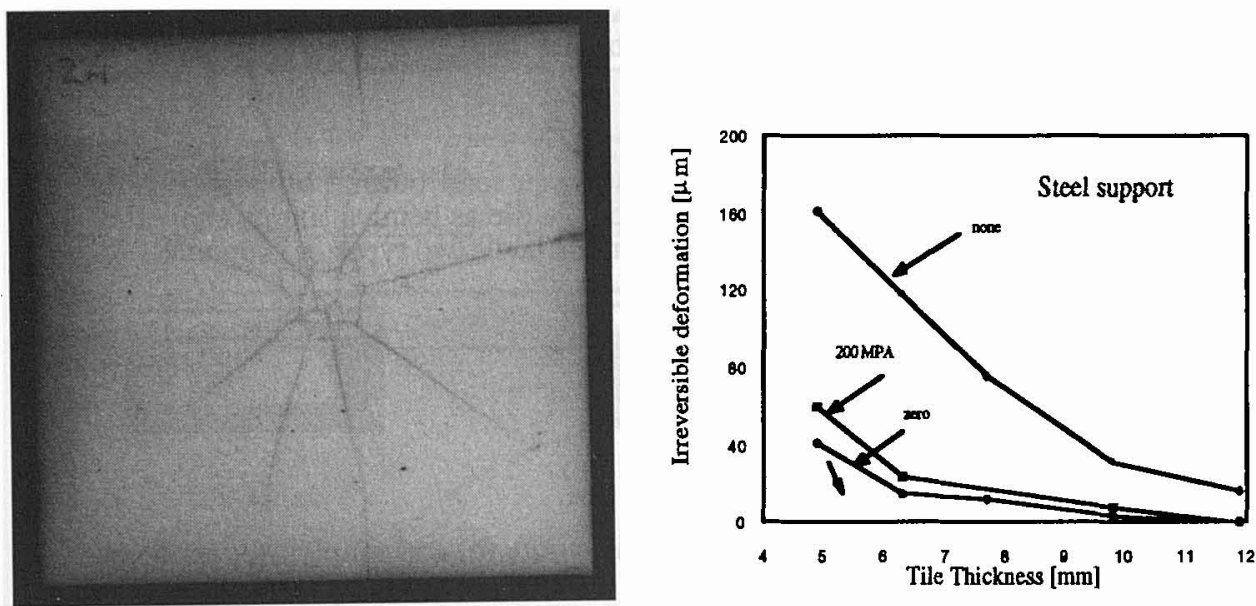

Fig. 4. Radial cracks in a confined $10 \mathrm{~mm}$ thick tile.

Fig. 5. Irreversible deflection of steel support

\subsubsection{Spectra composite support}

Alumina tiles supported by Spectra composite demonstrated more complex damage mechanisms. The tile/support impedance mismatch is high, and the stress waves wee fully reflected. Incident and reflected stress waves generated high tensile stresses parallel to the tile surfaces, causing spall damage.

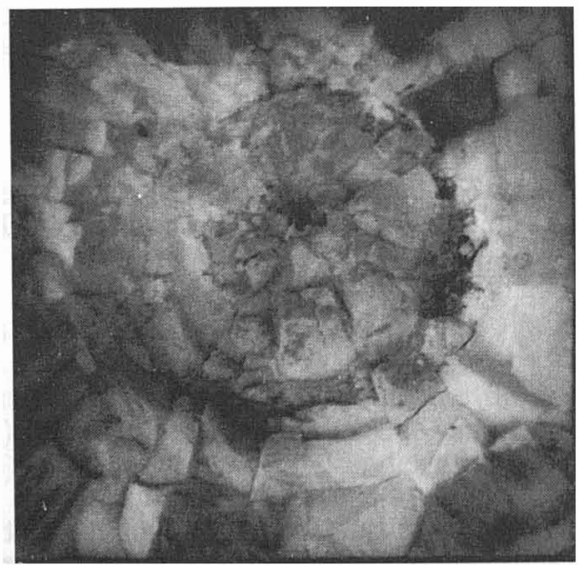

Fig. 6. Top view on the crater zone of tile supported by Spectra composite.

Indeed, the tiles, regardless of their thickness, were shattered to a small variety of fine fragments and alumina powder, define as a dynamic damage. That type of damage occurred in a circular zone of 30 $\mathrm{mm}$ diameter, matching the circular hole drilled in the cover plate. The reflected stress waves from the Spectra support continued to be reflected from the free (top) surface of the tile. The stress waves that met the cover plate dispersed and vanished without causing damage. A close view at the edge of the $30 \mathrm{~mm}$ crater showed thin layers of fractured alumina parallel to the tile surface (Fig. 6). An important phenomenon observed was the large number of radial crack patterns evident in the shattered tiles and which appeared in all the impacted tiles with no lateral confinement. 
Tiles with zero displacement at tile edges showed no change in the failure mechanism, all being shattered to fine fragments and powder. Quasi-static radial cracks were also observed. Tiles that were confined by $200 \mathrm{MPa}$ biaxial compressive stress suffered reduced quasi-static damage: no significant radial crack damage was observed. The dynamic damage due to stress waves reflected from the tile/support interface remained essentially the same.

\subsection{Finite width supports}

Tile/steel support assemblies of $6.5 / 6.5,8 / 8$, and $10 / 10 \mathrm{~mm}$ thickness were tested. The tiles were completely shattered, but the fragments were similar to those obtained for semi infinite support. The $6.5 \mathrm{~mm}$ thick steel supports had undergone massive plastic deformation and fractured by a single crack that crossed its bottom surface, resulted a large bending deformation. Less plastic deformation was observed in the $8 / 8 \mathrm{~mm}$ thick assembly, while the $10 / 10 \mathrm{~mm}$ thick assembly showed behavior similar to the semi infinite support.

The other type of tile/support assembly consisted of a fixed support of $6.5 \mathrm{~mm}$ thickness, while the tile thickness was varied. Tiles $6.5,8,10$, and $12 \mathrm{~mm}$ thick were fragmented to a large variety of fragments, but the nature of the fragments was similar to that observed for tiles supported by semi infinite steel. The same tile/support configurations were confined by zero displacement boundary condition. Reduced quasi-static damage was observed in the $6.5 \mathrm{~mm}$ tile, in addition to moderate dynamic damage in the form of spall cracks that sliced the tile through its thickness. The quasi-static and the dynamic damage were reduced in the thicker tiles, whereat the $12 \mathrm{~mm}$ thick tile the damage was dominated by quasi-static damage only.

Since the damage to tiles supported by semi infinite Spectra composite supports was dominated by dynamic damage, no tests were performed for tiles supported by finite width Spectra support.

\subsection{Simply supported tiles}

All the unconfined, simply supported tiles were shattered to a small variety of fine fragments and a large quantity of alumina powder. The massive damage was caused by the reflected stress waves generated in the tile upon impact. The same was observed in tiles confined by zero boundary condition.

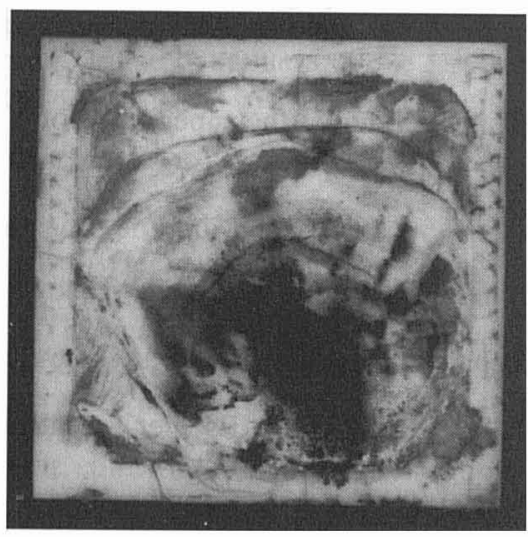

(a)

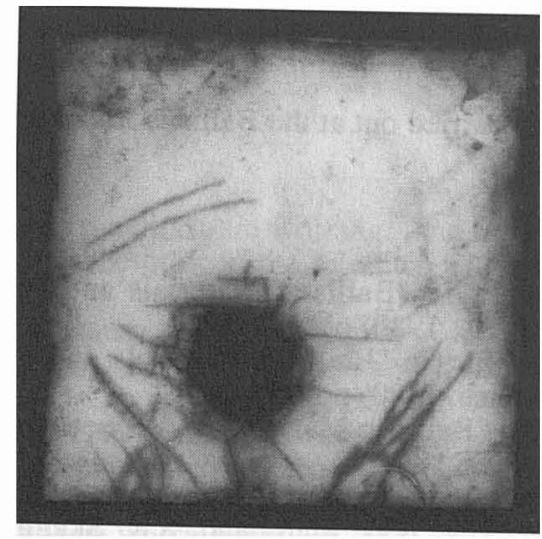

(b)

Fig. 7. The top (a) and bottom (b) surfaces of confined and simply supported alumina tile.

In the confined tiles, the damage changed dramatically. The tile remained in its original shape, and the top surface was pierced by a nearly circular hole of an average diameter of $10 \mathrm{~mm}$, Fig. 7a. Aside of several partially developed cracks, no other significant damage was observed in this surface. The bottom surface revealed a crater of large diameter and with rough surface, Fig. $7 \mathbf{b}$. The crater was shattered to fine fragments and alumina powder. 


\section{SUMMARY}

The damage to alumina tiles subjected to impact of 0.3 cal. NATO AP rounds was studied. Two types of damage mechanisms were identified: Quasi-static damage resulted from quasi static loads, while dynamic damage from reflected stress waves. The quasi-static damage is characterized by fragments of a large variety of sizes and shapes, while the dynamic damage is characterized by small variety of fine fragments to powder.

The quasi static damage was in the form of radial tensile cracks, initiated at the bottom surface of the tile and propagated to the top surface as a semi elliptical crack. Followed is a cone crack initiated at the tile/projectile contact zone as a pure mode II crack and propagated as a mixed mode crack. For sufficient kinetic energy, crushing of the cone envelope due to high compressive stresses terminates the damage mechanisms. The dynamic damage was in the form of spall cracks, generated by stress waves reflected from the tile/support interface.

While the semi infinite steel support caused no stress wave reflections, which have promoted the quasi-static damage, the Spectra composite caused almost full reflections, resulted predominant dynamic damage. Furthermore, changing the steel support thickness in order to promote stress wave reflection with varied tile thicknesses and levels of confinement caused only moderate changes in the damage mechanisms, so that the quasi static damage mechanisms still dominated.

The lateral confinement reduced the quasi-static damage significantly. A $12 \mathrm{~mm}$ thick tile on a semi infinite steel support block, subjected to $200 \mathrm{MPa}$ biaxial compressive stresses exhibited only 5 partially developed radial cracks. The effectiveness of confined alumina tiles in defeating a projectile was found to be considerably higher since the stress concentration at the tile/support interface reduced. Consequently, the tile is more effective in breaking the projectile due to delayed fracture. However, it appears that lateral confinement does not affect the dynamic damage, since impacted tiles on Spectra composite shattered into fine fragments regardless of the boundary condition and thickness.

The energy dissipated by the quasi-static damage mechanisms is very low, due to the low fracture energy of ceramics $\left(20-50 \mathrm{Jm}^{-2}\right)$ and small fracture surfaces. The energy dissipated by dynamic damage mechanisms may be two or more orders of magnitude higher, yet small compared to the overall kinetic energy of a common impactor.

\section{Acknowledgment}

AP tests were carried out at the Ballistic Laboratory of RAFAEL, Israel, under the supervision of $Y$. Rot.

\section{References}

[1] R.C. Laible, in "Ballistic Materials and Penetration Mechanics", Laible R.C. Ed. (Elsevier Scientific Publish. 1980) pp. 133-143.

[2] M.L. Wilkins, in "Ballistic Materials and Penetration Mechanics", Laible R.C. Ed. (Elsevier Scientific Publish. 1980) pp. 225-252

[3] H. Kolsky, "Ballistic Materials and Penetration Mechanics", Laible R.C. Ed. (Elsevier Scientific Publish. 1980) pp 185-222.

[4] D. Sherman and D.G. Brandon, In press, J. Mater. Research, 1997.

[5] D.A. Shockey, A.H. Marchand, S.R. Skaggs, G.E. Cort, M.W. Burkett and R. Parker, Int. J. Impact Eng. [9] (1990), pp. 263-275.

[6] D. Sherman and T. Ben-Shushan, Submitted.

[7] P. Riou, L. Beylat, C. Cottenot and J.L. Derep, J. de Physique IV, C8, No. 4, 1994, pp. $281-287$.

[8] D.L. Orphal, R.R. Franzen, A.J. Piekutowski, M.J. Forrestal, Int. J. Impact Engng., 18, 1996, pp.355-368.

[9] J. Krautkramer and H. Krautkramer, "Ultrasonic Testing of Materials", 4th Edition, SpringerVerlag, 1990. 